\title{
Leisure-Time Physical Activity and Metabolic Syndrome in Older Adults
}

\author{
Laura Gallardo-Alfaro ${ }^{1}$, Maria del Mar Bibiloni ${ }^{1}$, David Mateos ${ }^{1}$, Lucía Ugarriza ${ }^{1,2}$ and \\ Josep A. Tur ${ }^{1, * \mathbb{D}}$ \\ 1 Research Group on Community Nutrition \& Oxidative Stress, University of Balearic Islands, \\ IDISBA \&CIBEROBN (Physiopathology of Obesity and Nutrition), 07122 Palma de Mallorca, Spain; \\ lauragala3@gmail.com (L.G.-A.); mar.bibiloni@uib.es (M.d.M.B.); david-mateos@hotmail.es (D.M.); \\ luciaugarriza@gmail.com (L.U.) \\ 2 Camp Redó Primary Health Care Center, IBSalut, Palma de Mallorca, 07120 Palma de Mallorca, Spain \\ * Correspondence: pep.tur@uib.es; Tel.: +34-971-1731
}

Received: 25 July 2019; Accepted: 9 September 2019; Published: 11 September 2019

\begin{abstract}
Background: Metabolic syndrome (MetS) is a cluster of risk factors for cardiovascular disease, atherosclerosis and diabetes mellitus type 2 which may be reduced by practicing regular physical activity. Objective: To assess the leisure-time physical activity (LTPA) of older adults with MetS and without MetS. Methods: Cross-sectional study of older adults (55-80 years old) from Balearic Islands (Spain) with MetS ( $n=333 ; 55 \%$ men) and without MetS ( $n=144 ; 43.8 \%$ men). LTPA was assessed with the validated Spanish version of the Minnesota LTPA Questionnaire. Two criteria of physically active were used: $>150 \mathrm{~min} /$ week of moderate physical activity or $>75 \mathrm{~min} /$ week of vigorous physical activity or a combination of both, and total leisure-time energy expenditure of $>300 \mathrm{MET} \cdot \mathrm{min} /$ day. Sociodemographic and lifestyle characteristics, anthropometric variables, MetS components, and adherence to the Mediterranean diet (MD) were also measured. Results: MetS subjects showed lower energy expenditure in LTPA, lower adherence to the MD, higher obesity and waist circumference, and were less active than non-MetS peers. LTPA increased as participants got older and there was higher LTPA intensity as educational level increased. Adherence to MD was as high as LTPA was. Conclusions: MetS is associated with physical inactivity and unhealthy diet. To increase LTPA recommendations and raise awareness in the population about the health benefits of PA and high adherence to MD is highly recommended.
\end{abstract}

Keywords: metabolic syndrome; physical activity; leisure-time; older adults

\section{Introduction}

Metabolic syndrome (MetS) is a complex of interrelated risk factors for cardiovascular disease (CVD), atherosclerosis and diabetes mellitus type 2 (DM2) [1], responsible for two-fold increase in coronary heart disease risk, cerebrovascular disease risk, and 1.5-fold increase in all-cause mortality risk [2]. Risk factors include disglycaemia, high blood pressure, raised triglyceride levels, low high-density lipoprotein cholesterol levels, and obesity (particularly central adiposity) [2-5]. The causes responsible for the MetS seem to be multifactorial, including family history and lifestyle [6].

MetS prevalence is rising worldwide, which largely relates to an increasing inactive lifestyle, inadequate nutrition, ageing of population, and obesity [1,3]. As obesity prevalence doubled in the last three decades, MetS prevalence increased in parallel [7]. The average prevalence of MetS is nearly $35 \%$ of all adults and $46.7 \%$ of those aged 60 years or older [8]. MetS is now both a public health and clinical problem. 
Physical inactivity has been recognized as a global pandemic, representing one of the most pressing public health problems of the 21st century [9]. According to the World Health Organization (WHO), physical inactivity has been identified as the fourth leading risk factor for global mortality $[10,11]$. The evidence shows that practicing regular physical activity (PA), with at least $150 \mathrm{~min}$ of moderate-intensity aerobic PA per week or 75 min of vigorous-intensity aerobic PA per week, or an equivalent combination of moderate-and vigorous-intensity PA [10], improves insulin sensitivity, reduces hypertriglyceridemia, improves fibrinolytic ability, and decreases blood pressure as well as the risk of developing major cardiovascular and metabolic diseases [12,13]. In addition, exercise intensity is an important factor reversing the risk factors of the MetS [14]. Regular, moderate and vigorous PA has been demonstrated to prevent MetS [14-16]. Light PA enhances energy expenditure, which is associated with a lower prevalence of MetS [6]. Even a recent increase in PA level can have an important effect on health [17]. In this way, PA is related to healthy aging [18-20] and reduces the likelihood of developing MetS [21]. Consequently, an inactive lifestyle, in which people spend a large amount of their day sitting or lying down [22] is associated with increased mortality compared to a physically active lifestyle [23].

On the other hand, The Mediterranean Diet (MD) is characterized by a high consumption of fruits and nuts, vegetables, legumes and cereals, a high intake of olive oil as the principal source of dietary lipids, a low intake of saturated lipids and meat, a moderate intake of fish, a low-to-moderate intake of dairy products and a wine consumption in low to moderate amounts [24]. Furthermore, there is strong evidence that the adherence to a Mediterranean dietary pattern (MDP) is associated with a healthier status, due to the protective effect of MD against several chronic diseases, including CVD, several cancers and total mortality [24-27]. Moreover, adherence to MD has shown to be inversely associated with MetS [28,29].

In this way, practicing regular PA and high MD adherence seem to be important contributors to reduce the incidence of MetS. Therefore, the main objective of this study is to assess the leisure-time physical activity (LTPA) of older adults according to the presence or absence of MetS.

\section{Methodology}

\subsection{Study Design, Sample and Ethics}

This is a cross-sectional study to assess the effect of lifestyle factors on the health of older adults living in Balearic Islands, Spain. The study population was 477 participants, men (52\% of participants) aged 55-80 years and women aged 60-80 years with no previously documented cardiovascular disease engaged in social and municipal clubs, health centers, and sport clubs. Exclusion criteria included being institutionalized, suffering from a physical or mental illness which would have limited their participation in physical fitness assessment or their ability to respond to questionnaires, chronic alcoholism or drug addiction and intake of drugs for clinical research over the past year.

The study protocol and procedures were performed according to the ethical standards of the Declaration of Helsinki, and were approved by the Ethics Committee of Research of Balearic Islands (CEIC-IB2251/14PI and CEIC-IB1295/09PI). All participants provided written informed consent prior to participation.

\subsection{Socioeconomic and Lifestyle Determinants}

Sociodemographic and lifestyle characteristics were collected from each participant. Educational level was ranked into primary school studies, secondary school studies and university graduate. Civil status was ranked into single, married, divorced and widow/er. Finally, information related to individual medical history, current medication use and smoking status were also obtained. 


\subsection{Anthropometric Measurements}

Anthropometric variables were measured by trained personnel to minimize the inter-observer coefficients of variation. Height was measured using a wall-mounted stadiometer, to the nearest millimeter, with the subject's head in the Frankfurt plane. Weight was measured with high-quality electronic calibrated scales. Participants were weighed in bare feet and light clothes, subtracting $0.6 \mathrm{~kg}$ for their clothes. Body mass index (BMI) was calculated as weight in kilograms divided by the square of height in meters $\left(\mathrm{kg} / \mathrm{m}^{2}\right)$. Obesity was defined as BMI $\geq 30 \mathrm{~kg} / \mathrm{m}^{2}[2,30]$. Waist circumference (WC) was measured halfway between the last rib and the iliac crest by using an anthropometric tape. Blood pressure was measured with a validated semi-automatic oscillometer (Omron HEM-705CP) after $5 \mathrm{~min}$ of rest in-between measurements while the participant was in a seated position. All anthropometric variables were determined in duplicate, except for blood pressure (in triplicate).

\subsection{Metabolic Syndrome Classification}

Samples of fasting blood were collected from antecubital vein after an overnight fast. Biochemical analyses were performed on fasting plasma glucose, total cholesterol, low high-density lipoprotein cholesterol (HDL-c) and triglyceride (TG) concentrations in local laboratories using standard enzymatic methods. Participants were classified as "with MetS (MetS)" ( $n=333)$ and "without MetS (non-MetS)" $(n=144)$ according to the updated harmonized definition of the International Diabetes Federation and the American Heart Association and National Heart, Lung and Blood Institute [3].

\subsection{Mediterranean Diet Adherence}

The Mediterranean Dietary Pattern (MDP) was assessed according to a previously defined score indicating the degree of adherence to the traditional MD [25,31]. This Mediterranean dietary score (MDS) was converted to relative percentage of adherence using a previously described method [32,33] that is briefly summarized. An energy-adjusted value was obtained for each individual for the daily consumption of legumes, cereals (including bread and potatoes), fruit, vegetables, meat (including meat products), and milk (including dairy products). Information about the consumption of all these food items was obtained from the 137-item food-frequency questionnaire (FFQ), repeatedly validated in Spain [34]. For each item, a typical portion size was included and consumption frequencies were registered in 9 categories that ranged from "never or almost never" to " $\geq 6$ times/day". Energy and nutrient intakes were calculated as frequency multiplied by nutrient composition of specified portion size for each food item, using a self-made computerized program based on available information in Spanish food composition tables [35]. Monounsaturated fatty acids (MUFA):Saturated fatty acids (SFA) ratio was calculated. In order to score "moderate alcohol consumption", a transformation centred at the level of consuming $30 \mathrm{~g} /$ day for men (30-(30-absolute alcohol intake)), and $20 \mathrm{~g} /$ day for women (20-(20-absolute alcohol intake)) was used to obtain the highest value for men consuming $30 \mathrm{~g} /$ day or women consuming $20 \mathrm{~g} /$ day and progressive lower values as the consumption was lower or higher than these values. All these values were standardized as a $Z$ value [33,36]. Total MDS was computed by adding up all the $Z$ scores obtained for the favourable or more Mediterranean dietary components (legumes, cereals and roots, fruit, vegetables, fish, moderate alcohol, MUFA:SFA ratio) and subtracting the $Z$ value obtained from the consumption of meat and milk:

$$
\begin{gathered}
\sum Z=Z_{\text {legumes }}+Z_{\text {cereals and roots }}+Z_{\text {fruit }}+Z_{\text {vegetables }}+Z_{\text {fish }}+Z_{\text {moderate alcohol }}+Z_{\text {MUFA:SFA }} \\
-Z_{\text {meat }}-Z_{\text {milk }}
\end{gathered}
$$

The MDP was converted to relative percentage of adherence using the range of values of the sample. A participant with a maximum value of adherence in the sample obtained $100 \%$ of adherence. A participant with a minimum value of adherence obtained $0 \%$ in the relative percentage $[32,33]$. 


$$
\text { Adherence }\left(\text { Percentage }_{\mathrm{i}}\right)=\frac{\left(\sum \mathrm{Z}_{\mathrm{i}}-\sum \mathrm{Zmin}\right) * 100}{\left(\sum \mathrm{Zmax}-\sum \mathrm{Zmin}\right)}
$$

Once the percentage of adherence to the MDP was calculated, the variables that could determine a higher or lower adherence were assessed. Low Mediterranean Diet (MD) adherence was defined as a percentage of adherence below the 25th percentile, medium adherence was defined as a percentage of adherence between the 25th and 75th percentile and high adherence was defined as a percentage of adherence above the 75th percentile.

\subsection{Physical Activity}

PA was assessed using the validated Spanish version of the Minnesota Leisure Time Physical Activity Questionnaire [37,38], a valid instrument with good validity and reliability to measure the quantity and quality of PA performed in the last year [37-39]. Moreover, this questionnaire is considered a useful tool for estimating the amount of PA performed in elderly people [40,41]. This questionnaire, administered by interview with trained research assistants, measures leisure time physical activities (LTPA) including household activities, performed in the last year. This questionnaire was used to assess PA quality (light-moderate and vigorous) by using metabolic equivalents of task (MET) [42]. Light-moderate activity was defined as a MET intensity of PA lower and equal to 6 METs and vigorous activity was defined as a MET intensity of PA above 6 METs [43,44]. METs were calculated by multiplying the duration spent on that activity (measured in minutes) and their respective MET intensity levels. The MET score can be obtained from tables (the Compendium of Physical Activities) [44] that show the intensity of each activity relative to resting. MET-hour/week spent on PA refers to the energy expenditure that is spent on activities, over and above existing levels of resting energy expenditure.

\subsection{Criteria to Determine Active Population}

Two criteria were used to determine "physically active" population. First criterion is based on the "Global recommendations on PA for health" by the WHO. Therefore, active population was defined as practicing at least $150 \mathrm{~min}$ of moderate-intensity aerobic physical activity throughout the week, or doing at least $75 \mathrm{~min}$ of vigorous-intensity aerobic PA throughout the week, or an equivalent combination of moderate- and vigorous-intensity activity [10]. Lower PA was understood as inactive lifestyle. A second criterion established active population as expending above $300 \mathrm{MET} \cdot \mathrm{min} /$ day, which is related with lower heart attack risk $[45,46]$.

\subsection{Statistical Analyses}

Analyses were performed with the Statistical Package for the Social Sciences version 25.0 (IBM SPSS Statistics for Windows, Chicago, IL, USA). Categorical variables were presented as frequencies and/or proportions. Significant differences in prevalence were calculated by means of chi-squared test or Fisher's exact test. Continuous variables were presented as mean and standard deviation (SD) or median and interquartile range (IQR). Normality of data was assessed using Kolmogorov-Smirnov test. For normally distributed data, comparisons between two comparison groups were tested by unpaired Students' $t$-test. Equality of variances was assessed with Levene's test. For non-normally distributed data, the Mann-Whitney $U$ test was used to compare the median of two independent groups. Comparisons above 2 groups were tested by the Kruskal-Wallis test and/or the Mann Whitney $\mathrm{U}$ test, applying the Bonferroni correction. Correlations between LTPA and MD adherence were calculated using the Spearman rank correlation. Results were considered statistically significant if $p$-value ( 2 tailed) $<0.05$. 


\section{Results}

Table 1 shows general characteristics of MetS and non-MetS participants. MetS group showed higher weight, BMI and waist circumference, as well as higher percentage of men, obese participants and smokers. MetS group also showed lower adherence to the MD, compared with the non-MetS group.

Table 1. Baseline characteristics of study sample between MetS and non-MetS participants.

\begin{tabular}{|c|c|c|c|}
\hline General Characteristics & MetS $(n=333)$ & Non-MetS $(n=144)$ & $p$-Value \\
\hline Men, $n(\%)$ & $183(55.0)$ & $63(43.8)$ & 0.028 \\
\hline Age, $y$ & $64.9 \pm 5.4$ & $65.5 \pm 5.6$ & 0.342 \\
\hline Weight, kg & $86.2 \pm 14.0$ & $69.1 \pm 12.5$ & $<0.001$ \\
\hline Height, $\mathrm{cm}$ & $163.1 \pm 9.3$ & $162.4 \pm 8.8$ & 0.482 \\
\hline BMI, $\mathrm{kg} / \mathrm{m}^{2}$ & $32.4 \pm 3.87$ & $26.1 \pm 3.3$ & $<0.001$ \\
\hline Waist circumference, $\mathrm{cm}$ & $108.8 \pm 11.4$ & $85.4 \pm 10.9$ & $<0.001$ \\
\hline Obesity, $n(\%)$ & $238(94.8)$ & $13(5.2)$ & $<0.001$ \\
\hline Civil status, $n(\%)$ & & & 0.137 \\
\hline Single & $16(5.0)$ & $5(3.5)$ & \\
\hline Married & $251(77.7)$ & $101(70.1)$ & \\
\hline Divorced & $22(6.8)$ & $13(9.0)$ & \\
\hline Widow/er & $34(10.5)$ & $25(17.4)$ & \\
\hline Education level, $n(\%)$ & & & 0.179 \\
\hline Primary & $153(47.4)$ & $68(47.2)$ & \\
\hline Secondary & $104(32.2)$ & $50(34.7)$ & \\
\hline University & $66(20.4)$ & $26(18.1)$ & \\
\hline Mediterranean Diet Adherence, $n(\%)$ & & & $<0.001$ \\
\hline Low $(<\mathrm{p} 25)$ & $97(29.1)$ & $22(15.3)$ & \\
\hline Medium (p 25-p 75) & $167(50.2)$ & $72(50.0)$ & \\
\hline $\operatorname{High}(>\mathrm{p} 75)$ & $69(20.7)$ & $50(37.7)$ & \\
\hline Smoker, $n(\%)$ & $46(13.9)$ & $9(6.3)$ & 0.017 \\
\hline
\end{tabular}

Abbreviations: BMI: body mass index; MetS: Metabolic Syndrome; p: percentile. Difference in means between MetS and without MetS group were tested by unpaired Students' $t$ test for normally distributed variables and by the Mann-Whitney U test for non-normally distributed data. Differences in percentages were tested by chi-squared test. Significant differences have been highlighted in bold.

Comparison of the energy expenditure in LTPA between MetS and non-MetS participants is shown in Table 2. There were differences between MetS and non-MetS subjects in total energy expenditure in LTPA in all variables, except for single and obese participants. In non-MetS participants there were differences in total activity in BMI, which was also observed in vigorous activity besides civil status, age and gender. In light-moderate activity there were differences in gender, age and educational level. In MetS group there were differences in total activity in age, educational level and MD adherence, which were also observed in light-moderate PA. In vigorous PA there were differences in gender, education level MD adherence and BMI. Furthermore, in MetS group, total LTPA was correlated with MD adherence $(r=0.214, p<0.001)$, but not in non-MetS group $(r=0.008, p=0.925)$.

Percentage of active and inactive population in MetS and non-MetS according to two criteria is shown in Table 3. MetS participants were less active than non-MetS. Considering the first criterion for active population ( $>150 \mathrm{~min} /$ week of moderate PA or $>75 \mathrm{~min} /$ week of vigorous PA), there were differences between MetS and non-MetS in gender, married, 55-64 year-old, primary and secondary education, medium MD adherence and non-obesity $\left(<30 \mathrm{~kg} / \mathrm{m}^{2}\right)$ participants. In addition, there were higher percentages of active participants as age increased. According to the second criterion ( $>300 \mathrm{MET}$ $\mathrm{min} /$ day), there were differences between MetS and non-MetS in all variables except for single and obese $\left(\geq 30 \mathrm{~kg} / \mathrm{m}^{2}\right)$ participants. Furthermore, in MetS group there were differences in percentage of active people according to age and adherence to MD. 
Table 2. Energy expenditure (MET hour/week) in leisure-time physical activity (LTPA) between MetS ( $n=333)$ vs. non-MetS $(n=144)$ participants by sex, age, civil status, education level, adherence to the Mediterranean diet (MD) and body mass index (BMI). Physical activity is divided in light-moderate activity ( $<6$ MET) and vigorous activity ( $\geq 6 \mathrm{MET})$.

\begin{tabular}{|c|c|c|c|c|c|c|c|c|c|c|c|c|c|c|c|}
\hline & \multicolumn{5}{|c|}{ Total Activity } & \multicolumn{5}{|c|}{ Light-Moderate Activity } & \multicolumn{5}{|c|}{ Vigorous Activity } \\
\hline & \multicolumn{2}{|c|}{ MetS } & \multicolumn{2}{|c|}{ Non-MetS } & \multirow{2}{*}{$p$-Value } & \multicolumn{2}{|c|}{ MetS } & \multicolumn{2}{|c|}{ Non-MetS } & \multirow[b]{2}{*}{$p$-Value } & \multicolumn{2}{|c|}{ MetS } & \multicolumn{2}{|c|}{ Non-MetS } & \multirow[b]{2}{*}{$p$-Value } \\
\hline & Mean \pm SD & $\begin{array}{c}\text { Median } \\
\text { (IQR) }\end{array}$ & Mean \pm SD & $\begin{array}{l}\text { Median } \\
\text { (IQR) }\end{array}$ & & Mean \pm SD & $\begin{array}{l}\text { Median } \\
\text { (IQR) }\end{array}$ & Mean \pm SD & $\begin{array}{l}\text { Median } \\
\text { (IQR) }\end{array}$ & & Mean \pm SD & $\begin{array}{c}\text { Median } \\
\text { (IQR) }\end{array}$ & Mean \pm SD & $\begin{array}{l}\text { Median } \\
\text { (IQR) }\end{array}$ & \\
\hline $\begin{array}{c}\text { Total } \\
\text { Gender }\end{array}$ & $60.5 \pm 48.0$ & $45.9(63.0)$ & $103.1 \pm 140.1$ & $83.9(46.2)$ & $<0.001$ & $56.7 \pm 47.2$ & $43.9(60.3)$ & $84.6 \pm 136.4$ & $68.6(42.3)$ & $<0.001$ & $3.8 \pm 9.2$ & $0.9(4.3)$ & $18.5 \pm 27.9$ & $9.0(17.6)$ & $<0.001$ \\
\hline Men & $60.8 \pm 49.9$ & $45.6(60.8)$ & $122.8 \pm 207.5$ & $83.5(57.1)$ & $<0.001$ & $55.7 \pm 49.0$ & $42.3(59.8)$ & $93.4 \pm 203.9^{1}$ & $61.4(54.3)$ & 0.005 & $5.1 \pm 11.7^{1}$ & $1.9(4.7)$ & $29.4 \pm 37.7^{1}$ & $10.1(34.8)$ & $<0.001$ \\
\hline Women & $60.2 \pm 45.7$ & $46.0(62.9)$ & $87.7 \pm 34.0$ & $84.3(44.3)$ & $<0.001$ & $57.8 \pm 45.1$ & $45.6(60.6)$ & $77.6 \pm 30.6^{1}$ & $73.4(38.4)$ & $<0.001$ & $2.3 \pm 4.1^{1}$ & $0.9(3.1)$ & $10.1 \pm 11.2^{1}$ & $4.7(13.1)$ & $<0.001$ \\
\hline $\begin{array}{l}55-64 \\
65-69 \\
\geq 70\end{array}$ & $\begin{array}{c}49.6 \pm 41.8^{\mathrm{a}, \mathrm{b}} \\
73.6 \pm 50.7^{\mathrm{a}} \\
66.2 \pm 52.1^{\mathrm{b}}\end{array}$ & $\begin{array}{l}36.9(59.3) \\
59.6(73.3) \\
51.3(50.8)\end{array}$ & $\begin{array}{c}120.1 \pm 204.9 \\
88.5 \pm 43.3 \\
90.8 \pm 35.9\end{array}$ & $\begin{array}{l}86.2(61.7) \\
81.2(43.1) \\
82.8(42.5)\end{array}$ & $\begin{array}{c}<0.001 \\
0.037 \\
<0.001\end{array}$ & $\begin{array}{c}45.7 \pm 40.3^{\mathrm{a}, \mathrm{b}} \\
69.2 \pm 49.7^{\mathrm{a}} \\
63.4 \pm 52.6^{\mathrm{b}}\end{array}$ & $\begin{array}{c}\text { Age } \\
34.4(58.7) \\
52.7(68.6) \\
48.0(46.8)\end{array}$ & $\begin{array}{c}92.0 \pm 201.6^{\mathrm{b}} \\
75.5 \pm 35.9 \\
82.8 \pm 33.5^{\mathrm{b}}\end{array}$ & $\begin{array}{l}60.0(43.2) \\
75.6(37.3) \\
76.5(38.5)\end{array}$ & $\begin{array}{l}<0.001 \\
0.157 \\
<0.001\end{array}$ & $\begin{array}{l}3.9 \pm 10.7 \\
4.4 \pm 8.6 \\
2.8 \pm 5.7\end{array}$ & $\begin{array}{l}1.4(4.6) \\
1.4(4.7) \\
0.9(2.9)\end{array}$ & $\begin{array}{c}28.1 \pm 36.8^{\mathrm{b}} \\
13.0 \pm 17.0 \\
8.0 \pm 7.8^{\mathrm{b}}\end{array}$ & $\begin{array}{l}10.2(33.6) \\
5.4(16.7) \\
5.0(12.5)\end{array}$ & $\begin{array}{l}<0.001 \\
<0.001 \\
<0.001\end{array}$ \\
\hline & & & & & & & Civil status & & & & & & & & \\
\hline Single & $54.5 \pm 33.5$ & $43.69(50.3)$ & $384.4 \pm 723.0$ & $71.9(848.6)$ & 0.445 & $53.0 \pm 33.2$ & $43.7(48.4)$ & $371.0 \pm 715.8$ & $62.9(824.8)$ & 0.612 & $1.6 \pm 2.0$ & $0.5(4.3)$ & $13.4 \pm 15.5$ & $5.2(29.0)$ & 0.066 \\
\hline Married & $64.1 \pm 50.5$ & $51.2(62.1)$ & $96.7 \pm 50.5$ & $88.4(48.1)$ & $<0.001$ & $59.8 \pm 49.7$ & $45.0(60.6)$ & $76.1 \pm 39.0$ & & $<0.001$ & $4.3 \pm$ & 1.4 & $20.6 \pm 28.5^{c}$ & 2.4) & $<0.001$ \\
\hline Divorced & $39.5 \pm 31.9$ & $26.1(64.8)$ & $93.8 \pm 38.7$ & $87.9(56.1)$ & $<0.001$ & $36.4 \pm 31.0$ & $22.7(63.0)$ & $65.7 \pm 30.2$ & $59.2(5$ & 0.009 & 3.1 & $2.1(4.7)$ & $28.1 \pm 41.6$ & $15.8(33.3)$ & 0.005 \\
\hline Widow/er & $50.5 \pm 38.9$ & $41.2(70.1)$ & $77.6 \pm 32.2$ & $67.4(46.2)$ & 0.001 & $48.6 \pm 39.1$ & $\begin{array}{c}36.0(71.8) \\
\end{array}$ & $71.2 \pm 30.9$ & $65.1(32.4)$ & 0.003 & $1.9 \pm 3.1$ & $0.7(2.9)$ & $6.3 \pm 10.0^{c}$ & $3.7(3.6)$ & 0.002 \\
\hline Primary & $68.5 \pm 51.4^{\mathrm{d}}$ & $57.0(69.9)$ & $100.4 \pm 54.5$ & $94.2(46.6)$ & $<0.001$ & $65.8 \pm 50.9^{d}$ & $54.6(71.3)$ & $81.7 \pm 37.4 \mathrm{e}$ & $78.0(45.7)$ & 0.001 & $2.7 \pm 5.3^{\mathrm{d}}$ & $0.9(2.8)$ & 3.5 & $7.6(13.2)$ & $<0.001$ \\
\hline Secondary & $58.2 \pm 45.8$ & 44.7 (60.1) & $115.1 \pm 228.3$ & 73.4 (48.2) & $<0.001$ & $53.9 \pm 44.3$ & $40.6(56.1)$ & $96.9 \pm 226.9^{\mathrm{e}}$ & $58.5(44.7)$ & 0.01 & $4.3 \pm 13.1 \mathrm{f}$ & $1.2(4.6)$ & $18.2 \pm 24.4$ & $6.6(25.8)$ & $<0.001$ \\
\hline University & $42.7 \pm 30.5^{\mathrm{d}}$ & $36.5(49.4)$ & $87.0 \pm 39.8$ & $85.7(49.6)$ & $<0.001$ & $36.7 \pm 26.8^{\mathrm{d}}$ & 31.1 (34.7) & $68.3 \pm 34.8$ & $62.1(40.4)$ & $<0.001$ & $6.0 \pm 9.7 \mathrm{df}$ & $3.3(6.6)$ & $18.8 \pm 16.7$ & $13.2(29.4)$ & $<0.001$ \\
\hline Low $(<\mathrm{p} 25)$ & $\pm 37.9^{\mathrm{i}, \mathrm{k}}$ & 33 & $170.3 \pm 340.9$ & 82.1 & $<0.00$ & $38.5 \pm 36.5^{\mathrm{i}, \mathrm{k}}$ & $\begin{array}{c}\text { rranean Diet Ac } \\
28.7(40.4)\end{array}$ & $\begin{array}{l}\text { therence } \\
453.2 \pm 337.3\end{array}$ & 66.1 & $<0.001$ & $3.9 \pm 1$ & 4) & 17 & 7.0( & $<0.001$ \\
\hline Medium (p 50-75) & $65.8 \pm 48.2^{i, j}$ & $53.6(67.2)$ & $89.9 \pm 47.0$ & $86.1(42.0)$ & $<0.001$ & $62.1 \pm 47.8^{\mathrm{i}, \mathrm{j}}$ & $50.7(64.5)$ & $71.8 \pm 37.6$ & $69.2(39.5)$ & 0.023 & $\pm 7.2^{\mathrm{i}, \mathrm{j}}$ & $0.9(4.4)$ & $18.1 \pm 26.9$ & $9.4(19.6)$ & $<0.001$ \\
\hline $\begin{array}{c}\text { High }(\mathrm{p}>75) \\
\text { BMI }\end{array}$ & $72.0 \pm 53.5^{\mathrm{k}, \mathrm{j}}$ & $58.6(76.2)$ & $92.5 \pm 43.5$ & $82.8(52.9)$ & 0.004 & $67.8 \pm 52.2^{\mathrm{k}, \mathrm{j}}$ & $54.6(72.1)$ & $72.7 \pm 32.1$ & $70.6(54.4)$ & 0.137 & $4.1 \pm 6.5^{\mathrm{k}, \mathrm{j}}$ & $2.3(4.7)$ & $19.8 \pm 31.2$ & $9.4(17.1)$ & $<0.001$ \\
\hline$<30 \mathrm{~kg} / \mathrm{m} 2$ & $59.2 \pm 38.9$ & $48.3(50.5)$ & $106.4 \pm 145.9 \mathrm{~g}$ & $88.0(46.4)$ & $<0.001$ & $53.7 \pm 37.0$ & $43.9(54.3)$ & $87.0 \pm 142.5$ & $68.7(43.6)$ & $<0.001$ & $5.5 \pm 8.7^{\mathrm{h}}$ & $2.8(5.7)$ & $19.4 \pm 28.6^{g}$ & $9.4(17.5)$ & $<0.001$ \\
\hline$\geq 30 \mathrm{~kg} / \mathrm{m} 2$ & $61.1 \pm 51.6$ & 45.5 (68.6) & $69.8 \pm 45.3 \mathrm{~g}$ & $65.3(34.5)$ & 0.253 & $58.0 \pm 51.1$ & $43.8(67.1)$ & $60.0 \pm 35.3$ & $61.7(43.3)$ & 0.407 & $3.1 \pm 9.3^{\mathrm{h}}$ & $0.9(2.8)$ & $9.8 \pm 17.4 \mathrm{~g}$ & $1.1(10.6)$ & 0.178 \\
\hline
\end{tabular}

Abbreviations: BMI: body mass index; IQR: Interquartil range; MD: Mediterranean Diet; MET: Metabolic equivalent; MetS: Metabolic Syndrome; p: percentile; SD: Standard deviation.

Difference in means for intensity of physical activity (total physical activity, light-moderate activity, heavy activity) between MetS and non-MetSwere tested by the Mann Whitney U test. Differences among categories of the same group were tested by the Kruskal-Wallis test and/or the Mann Whitney U test, applying the Bonferroni correction. ${ }^{a}$ Differences ( $p$-value $<0.05$ ) between 55-64 years vs. 65-69 years old in the same group (MetS). ${ }^{\mathrm{b}}$ Differences ( $p$-value $<0.05$ ) between $55-64$ years vs. $\geq 70$ years old in the same group (non-MetS or MetS). ${ }^{c}$ Differences ( $p$-value $<0.05)$ between married vs. widow/er in the same group (non-MetS). ${ }^{d}$ Differences ( $p$-value $\left.<0.05\right)$ between primary studies vs. university studies in the same group (MetS). ${ }^{\mathrm{e}}$ Differences ( $p$-value $<0.05$ ) between primary studies vs. secondary studies in the same group (non-MetS). ${ }^{\mathrm{f}}$ Differences $(p$-value $<0.05)$ between primary studies vs. university studies in the same group (MetS). ${ }^{g}$ Differences ( $p$-value $<0.05$ ) between $<30 \mathrm{~kg} / \mathrm{m}^{2} \mathrm{vs} . \geq 30 \mathrm{~kg} / \mathrm{m}^{2}$ in the same group (non-MetS). ${ }^{\mathrm{h}}$ Differences $(p$-value $<0.001) \mathrm{between}<30 \mathrm{~kg} / \mathrm{m}^{2} \mathrm{vs.} \geq 30 \mathrm{~kg} / \mathrm{m}^{2}$ in the same group (MetS). ${ }^{\mathrm{i}}$ Differences ( $p$-value $<0.05$ ) between low MD adherence vs. medium MD adherence in the same group (MetS). ${ }^{j}$ Differences ( $p$-value $<0.05$ ) between high MD adherence vs. medium MD adherence in the same group (MetS). ${ }^{k}$ Differences ( $p$-value $<0.05$ ) between low MD adherence vs. high MD adherence in the same group (MetS). ${ }^{1}$ Differences between gender in the same group. Significant differences have been highlighted in bold. 
Table 3. Percentage of active and inactive population in MetS $(n=288)$ and non-MetS $(n=142)$ participants according to two criteria $(1$ st Criterion: $>150$ min/week of moderate physical activity or $>75 \mathrm{~min} /$ week of vigorous physical activity; 2nd Criterion: $>300 \mathrm{MET} \mathrm{min} /$ day).

\begin{tabular}{|c|c|c|c|c|c|c|c|c|c|c|}
\hline & \multicolumn{5}{|c|}{ 1st Criterion } & \multicolumn{5}{|c|}{ 2nd Criterion } \\
\hline & \multicolumn{2}{|c|}{ MetS $(n=333)$} & \multicolumn{2}{|c|}{ Non-MetS $(n=144)$} & \multirow{2}{*}{$p$-Value } & \multicolumn{2}{|c|}{ MetS $(n=333)$} & \multicolumn{2}{|c|}{ Non-MetS $(n=144)$} & \multirow{2}{*}{$p$-Value } \\
\hline & Active & Inactive & Active & Inactive & & Active & Inactive & Active & Inactive & \\
\hline Total & $257(89.2)$ & $31(10.8)$ & $142(100)$ & $0(0)$ & $<0.001$ & $180(62.5)$ & $108(37.5)$ & $134(93.1)$ & $10(6.9)$ & $<0.001$ \\
\hline \multicolumn{11}{|l|}{ Gender } \\
\hline Men & $139(88.5)$ & $18(11.5)$ & $62(100)$ & $0(0)$ & 0.002 & $96(61.1)$ & $61(38.9)$ & 57 (90.5) & $6(9.5)$ & $<0.001$ \\
\hline Women & $118(90.1)$ & $13(9.9)$ & $80(100)$ & $0(0)$ & 0.002 & $84(64.1)$ & 47 (35.9) & 77 (95.1) & $4(4.9)$ & $<0.001$ \\
\hline$p$-value & \multicolumn{2}{|c|}{0.675} & \multicolumn{2}{|c|}{1.000} & & \multicolumn{2}{|c|}{0.603} & \multicolumn{2}{|c|}{0.283} & \\
\hline \multicolumn{11}{|l|}{ Age } \\
\hline $55-64$ & $117(84.2)$ & $22(15.8)$ & $62(100)$ & $0(0)$ & $<0.001$ & $71(51.4)$ & $67(48.6)$ & $59(92.2)$ & $5(7.8)$ & $<0.001$ \\
\hline $65-69$ & $82(93.2)$ & $6(6.8)$ & $46(100)$ & $0(0)$ & 0.094 & $65(73.9)$ & $23(26.1)$ & $42(91.3)$ & $4(8.7)$ & 0.022 \\
\hline$\geq 70$ & $58(95.1)$ & $3(4.9)$ & $34(100)$ & $0(0)$ & 0.550 & $44(71.0)$ & $18(29.0)$ & 33 (97.1) & $1(2.9)$ & 0.002 \\
\hline$p$-value & \multicolumn{2}{|c|}{0.026} & \multicolumn{2}{|c|}{1.000} & & \multicolumn{2}{|c|}{0.001} & \multicolumn{2}{|c|}{0.567} & \\
\hline \multicolumn{11}{|l|}{ Civil status } \\
\hline Single & $14(93.3)$ & $1(6.7)$ & $5(100)$ & $0(0)$ & 1.000 & $10(66.7)$ & $5(33.3)$ & $4(80.0)$ & $1(20.0)$ & 1.000 \\
\hline Married & $200(89.7)$ & $23(10.3)$ & $99(100)$ & $0(0)$ & $<0.001$ & $145(65.0)$ & $78(35.0)$ & $93(92.1)$ & $8(7.9)$ & $<0.001$ \\
\hline Divorced & $16(80.0)$ & $4(20.0)$ & $13(100)$ & $0(0)$ & 0.136 & $7(35.0)$ & $13(65.0)$ & $13(100)$ & $0(0)$ & $<0.001$ \\
\hline Widow/er & $27(90.0)$ & $3(10.0)$ & $25(100)$ & $0(0)$ & 0.242 & $18(60.0)$ & $12(40.0)$ & $24(96.0)$ & $1(4.0)$ & 0.003 \\
\hline$p$-value & \multicolumn{2}{|c|}{0.551} & \multicolumn{2}{|c|}{1.000} & & \multicolumn{2}{|c|}{0.064} & \multicolumn{2}{|c|}{0.428} & \\
\hline \multicolumn{11}{|c|}{ Education level } \\
\hline Primary & $120(90.2)$ & $13(9.8)$ & $66(100)$ & $0(0)$ & 0.005 & $90(67.7)$ & 43 (32.3) & $62(91.2)$ & $6(8.8)$ & $<0.001$ \\
\hline Secondary & 79 (91.9) & $7(8.1)$ & $50(100)$ & $0(0)$ & 0.047 & 53 (61.6) & $33(38.4)$ & 48 (96.0) & $2(4.0)$ & $<0.001$ \\
\hline University & $50(84.7)$ & $9(15.3)$ & $26(100)$ & $0(0)$ & 0.052 & $30(50.8)$ & $29(49.2)$ & $24(92.3)$ & $2(7.7)$ & $<0.001$ \\
\hline$p$-value & \multicolumn{2}{|c|}{0.365} & \multicolumn{2}{|c|}{1.000} & & \multicolumn{2}{|c|}{0.085} & \multicolumn{2}{|c|}{0.587} & \\
\hline
\end{tabular}


Table 3. Cont

\begin{tabular}{|c|c|c|c|c|c|c|c|c|c|c|}
\hline & \multicolumn{5}{|c|}{ 1st Criterion } & \multicolumn{5}{|c|}{ 2nd Criterion } \\
\hline & \multicolumn{2}{|c|}{ MetS $(n=333)$} & \multicolumn{2}{|c|}{ Non-MetS $(n=144)$} & \multirow{2}{*}{$p$-Value } & \multicolumn{2}{|c|}{ MetS $(n=333)$} & \multicolumn{2}{|c|}{ Non-MetS $(n=144)$} & \multirow{2}{*}{$p$-Value } \\
\hline & Active & Inactive & Active & Inactive & & Active & Inactive & Active & Inactive & \\
\hline \multicolumn{11}{|c|}{ Mediterranean Diet Adherence } \\
\hline Low $(p<25)$ & $68(84.0)$ & $13(16.0)$ & $22(100)$ & $0(0)$ & 0.065 & $38(47.5)$ & $42(52.5)$ & $21(95.5)$ & $1(4.5)$ & $<0.001$ \\
\hline Medium (p 25-p 75) & $133(90.5)$ & $14(9.5)$ & $70(100)$ & $0(0)$ & 0.006 & $99(66.9)$ & $49(33.1)$ & $66(91.7)$ & $6(8.3)$ & $<0.001$ \\
\hline $\operatorname{High}(\mathrm{p}>75)$ & $56(93.3)$ & $4(6.7)$ & $50(100)$ & $0(0)$ & 0.084 & $43(71.7)$ & $17(28.3)$ & $47(94.0)$ & $3(6.0)$ & 0.003 \\
\hline$p$-value & \multicolumn{2}{|c|}{0.162} & \multicolumn{2}{|c|}{1.000} & & \multicolumn{2}{|c|}{0.004} & \multicolumn{2}{|c|}{0.787} & \\
\hline \multicolumn{11}{|l|}{ BMI } \\
\hline$<30 \mathrm{~kg} / \mathrm{m}^{2}$ & $82(92.1)$ & $7(7.9)$ & $129(100)$ & $0(0)$ & 0.002 & $61(69.3)$ & $27(30.7)$ & $123(93.9)$ & $8(6.1)$ & $<0.001$ \\
\hline$\geq 30 \mathrm{~kg} / \mathrm{m}^{2}$ & 175 (87.9) & 24 (12.1) & $13(100)$ & $0(0)$ & 0.370 & $119(59.5)$ & $81(40.5)$ & $11(84.6)$ & $2(15.4)$ & 0.084 \\
\hline$p$-value & \multicolumn{2}{|c|}{0.288} & \multicolumn{2}{|c|}{1.000} & & \multicolumn{2}{|c|}{0.113} & \multicolumn{2}{|c|}{0.209} & \\
\hline
\end{tabular}

Abbreviations: BMI: body mass index; MD: Mediterranean Diet; MetS: Metabolic Syndrome; p: percentile. Data is shown as $n$ (\%). Differences in percentages between MetS and non-MetS participants and in different variables of the same group were tested by chi-squared test or Fisher's exact test. Significant differences have been highlighted in bold. 


\section{Discussion}

The main finding of this study was the low energy expenditure in LTPA of MetS participants on most studied outcomes. LTPA increased as participants got older and there was higher LTPA intensity as educational level increased. MetS subjects also showed lower adherence to the MDP than non-MetS peers; and adherence to MD was as high as LTPA was.

Practicing regular PA is indicative of greater longevity and reduced risk of coronary heart disease, CVD, stroke and colon cancer [47]. LTPA reduces all causes and CVD death risk [48], as well as MetS development. According to the second criterion, it is important to note that adherence to MD increased in the MetS group as much as the energy expenditure in LTPA and the percentage of active participants did. This result was consistent with a previous study in which as total LTPA increased, the adherence to MD did too [49]. Furthermore, it was found that older adults with unhealthy diet tended to engage in more inactive lifestyle than older adults with a healthy diet [50].

Age is also an outcome to be considered. MetS participants who were 65-69 years old showed higher light-moderate PA levels than those aged 55-64 years. A plausible explanation could be that older people were retired, and they had more time to spend outside, increasing LTPA. This finding is contrary to a previous study pointing out that older participants were less active [51]. MetS subjects in our study showed higher levels of light-moderate PA, but lower vigorous PA than a previous study in Spain [52].

Despite that total and light-moderate PA of MetS subjects showed no differences between sexes, vigorous PA of male MetS subjects showed higher LTPA. No differences were found between sexes in percentage of physically active participants for both criteria, which is contrary to previous findings in which females were less active than men for LTPA [51].

Regarding educational level, MetS participants with university studies showed higher vigorous PA levels, which is related to lower mortality risk [53]. However, higher light-moderate PA levels were observed in MetS participants with primary and secondary education. As educational level increased, LTPA intensity did. Vigorous LTPA has been associated with lower risk of developing MetS [54]. Moreover, it was concluded that lower education level increased the incidence of mortality from cardiovascular disease [55]. Another previous study concluded that lower education was related with decreased LTPA among older adults [56]. On the contrary, our study showed no differences in percentage of active people in both groups regarding educational level, suggesting that as educational level increased, the LTPA intensity did, but not the percentage of active people.

Obesity is associated with numerous comorbidities such as CVD, type 2 diabetes, hypertension and several cancers $[57,58]$. Our results showed that MetS and non-MetS obese subjects showed no differences in energy expenditure in LTPA and in percentage of active population. These results were consistent with previous findings pointing out that higher inactive leisure time and lower PA were associated with greater prevalence of increased BMI [59], as well as that insufficient LTPA was related with higher BMI [60].

MetS subjects showed low adherence to the MD compared with non-MetS participants. It is important to highlight that MD is characterized by high consumption of vegetables, legumes, fruits and nuts and whole cereals, high intake of olive oil, but low-to-moderate consumption of dairy products, low intake of meat and poultry and regular, but moderate intake of wine [31,36,61]. Accordingly, MD is recommended to improve glycaemia and cardiovascular risk factors [62], with protective effect on cardiovascular diseases [63], also being a possible therapy for MetS [64]. It was previously concluded that as adherence to MD decreases, MetS increases, resulting in a worse profile of plasma inflammation markers [36]. Moreover, lower MD adherence was observed among patients with MetS, as well as an inverse association between adherence to MD and prevalence of MetS components [65].

In our study, physically active population, MetS subjects were more inactive than non-MetS peers after the two WHO criteria were considered [10]. According to the first criterion for active population ( $>150 \mathrm{~min} /$ week of moderate PA or $>75 \mathrm{~min} /$ week of vigorous PA or a combination of both), the MetS group showed $89.2 \%$ active participants, in contrast with $100 \%$ in the non-MetS group. 
Our results agree a previous study using the same recommendations, but although LTPA increased, overweight or obesity increased and physical fitness decreased [66]. Considering the second criterion (>300 MET·min/day) [46], there were 62.5\% MetS active participants and 93.1\% non-MetS active participants; however, our study reported higher percentage of active people among MetS participants than previous related studies [46,67].

\section{Strengths and Limitations}

The main strength of this study was that, to our knowledge, it is the first study comparing LTPA in older adults with and without MetS. However, the study has several limitations. First, this cross-sectional study limits the ability to elucidate a causal relationship between MetS and lower LTPA. Second, given that with the LTPA it is not possible to measure occupational activity, comparison of total physical activity among age groups is also limited. Finally, it is a quasi-experimental design.

\section{Conclusions}

MetS subjects showed lower energy expenditure in LTPA, were less active, with lower adherence to the MD and higher obesity and waist circumference than non-MetS peers. MetS is then associated to physical inactivity and unhealthy diet. To increase LTPA recommendations and raise awareness in the population about the health benefits of PA and high adherence to MD is highly recommended.

Author Contributions: Conceptualization, J.A.T. and M.d.M.B.; methodology, L.G.-A. and M.d.M.B.; validation, M.d.M.B. and J.A.T.; formal analysis, L.G.-A.; investigation, L.G.-A., D.M., L.U.; resources, J.A.T.; data curation, M.d.M.B.; writing—original draft preparation, L.G.-A. and M.d.M.B.; writing—review and editing, all authors; supervision, project administration and funding acquisition, J.A.T.

Funding: This study was supported by the official funding agency for biomedical research of the Spanish government, Institute of Health Carlos III(ISCIII) through the Fondo de Investigación para la Salud (FIS), which is co-funded by the European Regional Development Fund (Projects PI11/01791, PI14/00636 and PI17/01827, Red Predimed-RETIC RD06/0045/1004, and CIBEROBN CB12/03/30038), Grant of support to research groups no. 35/2011 (Balearic Islands Gov.), Fundació La Marató TV3 (Spain) project ref. 201630.10, and EU COST Action CA16112. The funders had no role in study design, data collection and analysis, decision to publish, or preparation of the manuscript.

Conflicts of Interest: The authors declare that they have no conflict of interest.

\section{References}

1. Moreira, G.C.; Cipullo, J.P.; Souza Ciorlia, L.A.; Bernardi Cesarino, C.; Vilela-Martin, J.F. Prevalence of metabolic syndrome: Association with risk factors and cardiovascular complications in an urban population. PLoS ONE 2014, 9, e105056. [CrossRef] [PubMed]

2. Engin, A. The definition and prevalence of obesity and metabolic syndrome. Adv. Exp. Med. Biol. 2017, 960, 1-17. [PubMed]

3. Alberti, K.G.; Eckel, R.H.; Grundy, S.M.; Zimmet, P.M.; Cleeman, J.I.; Donato, K.A.; Smith, S.C. Harmonizing the metabolic syndrome. Circulation 2009, 120, 1640-1645. [CrossRef] [PubMed]

4. Sherling, D.H.; Perumareddi, P.; Hennekens, C.H. Metabolic syndrome. J. Cardiovasc. Pharmacol. Ther. 2017, 22, 365-367. [CrossRef] [PubMed]

5. Oda, E. Historical perspectives of the metabolic syndrome. Clin. Dermatol. 2018, 36, 3-8. [CrossRef] [PubMed]

6. Serrano-Sánchez, J.A.; Fernández-Rodríguez, M.J.; Sanchis-Moysi, J.; Rodríguez-Pérez, M.D.C.; Marcelino-Rodríguez, I.; Cabrera de León, A. Domain and intensity of physical activity are associated with metabolic syndrome: A population-based study. PLoS ONE 2019, 14, e0219798. [CrossRef] [PubMed]

7. Samson, S.L.; Garber, A.J. Metabolic syndrome. Endocrinol. Metabol. Clin. N. Am. 2014, 43, 1-23. [CrossRef]

8. Aguilar, M.; Bhuket, T.; Torres, S.; Liu, B.; Wong, R.J. Prevalence of the metabolic syndrome in the United States, 2003-2012. J. Am. Med. Assoc. 2015, 313, 1973. [CrossRef]

9. Al-Tunaiji, H.; Davis, J.C.; Mansournia, M.A.; Khan, K.M. Population attributable fraction of leading non-communicable cardiovascular diseases due to leisure-time physical inactivity: A systematic review. BMJ Open Sport Exerc. Med. 2019, 5, e000512. [CrossRef] 
10. World Health Organization. Global Recommendations on Physical Activity for Health; WHO Press: Geneva, Switzerland, 2010. Available online: https://www.who.int/dietphysicalactivity/global-PA-recs-2010.pdf (accessed on 25 July 2019).

11. Kohl, H.W.; Craig, C.L.; Lambert, E.V.; Inoue, S.; Alkandari, J.R.; Leetongin, G.; Lancet Physical Activity Series Working Group. The pandemic of physical inactivity: Global action for public health. Lancet 2012, 380, 294-305. [CrossRef]

12. Lee, I.M.; Shiroma, E.J.; Lobelo, F.; Puska, P.; Blair, S.N.; Katzmarzyk, P.T.; Lancet Physical Activity Series Working Group. Effect of physical inactivity on major non-communicable diseases worldwide: An analysis of burden of disease and life expectancy. Lancet 2012, 380, 219-229. [CrossRef]

13. McPhee, J.S.; French, D.P.; Jackson, D.; Nazroo, J.; Pendleton, N.; Degens, H. Physical Activity in Older Age: Perspectives for healthy ageing and frailty. Biogerontology 2016, 17, 567-580. [CrossRef] [PubMed]

14. Tjønna, A.E.; Lee, S.J.; Rognmo, Ø.; Stølen, T.O.; Bye, A.; Haram, P.M. Aerobic Interval Training Versus Continuous Moderate Exercise as a Treatment for the Metabolic Syndrome. Circulation 2008, 118, 346-354. [CrossRef]

15. Katzmarzyt, P.T.; Leon, A.S.; Wilmore, J.H.; Skinner, J.S.; Rao, D.C.; Rankinen, T.; Bouchard, C. Targeting the Metabolic Syndrome with Exercise: Evidence from the HERITAGE Family Study. Med. Sci. Sports Exerc. 2003, 35, 1703-1709. [CrossRef] [PubMed]

16. Stewart, K.J.; Bacher, A.C.; Turner, K.; Lim, J.G.; Hees, P.S.; Shapiro, E.P.; Ouyang, P. Exercise and risk factors associated with metabolic syndrome in older adults. Am. J. Prev. Med. 2005, 28, 9-18. [CrossRef]

17. Westerterp, K.R. Daily physical activity and ageing. Curr. Opin. Clin. Nutr. Metabol. Care 2000, 3, 485-488. [CrossRef]

18. Menec, V.H. The relation between everyday activities and successful aging: A 6-year longitudinal study. J. Gerontol. Ser. B Psychol. Sci. Social Sci. 2003, 58, S74-S82. [CrossRef]

19. Harridge, S.D.R.; Lazarus, N.R. Physical activity, aging, and physiological function. Physiology 2017, 32, 152-161. [CrossRef]

20. Reas, E.T.; Laughlin, G.A.; Bergstrom, J.; Kritz-Silverstein, D.; Richard, E.L.; Barrett-Connor, E.; McEvoy, L.K. Lifetime physical activity and late-life cognitive function: The Rancho Bernardo study. Age Ageing 2019, 48, 241-246. [CrossRef]

21. Xu, F.; Cohen, S.A.; Lofgren, I.E.; Greene, G.W.; Delmonico, M.J.; Greaney, M.L. The Association between physical activity and metabolic syndrome in older adults with obesity. J. Frailty Aging 2019, 8, 27-32.

22. Magnon, V.; Dutheil, F.; Auxiette, C. Sedentariness: A Need for a Definition. Front. Public Health 2018, 6, 372. [CrossRef] [PubMed]

23. Westheim, A.; Os, I. Physical activity and the metabolic cardiovascular syndrome. J. Cardiovasc. Pharmacol. 1992, 20 (Suppl. S8), S49-S53. [CrossRef] [PubMed]

24. Serra-Majem, L.; Roman, B.; Estruch, R. Scientific evidence of interventions using the Mediterranean diet: A systematic review. Nutr. Rev. 2006, 64, S27-S47. [CrossRef] [PubMed]

25. Trichopoulou, A.; Kouris-Blazos, A.; Wahlqvist, M.L.; Gnardellis, C.; Lagiou, P.; Polychronopoulos, E.; Trichopoulos, D. Diet and overall survival in elderly people. Br. Med. J. 1995, 311, 1457-1460. [CrossRef] [PubMed]

26. Sofi, F.; Cesari, F.; Abbate, R.; Gensini, G.F.; Casini, A. Adherence to Mediterranean diet and health status: Meta-analysis. Br. Med. J. 2008, 337, a1344. [CrossRef] [PubMed]

27. Grosso, G.; Mistretta, A.; Frigiola, A.; Gruttadauria, S.; Biondi, A.; Basile, F.; Galvano, F. Mediterranean Diet and cardiovascular risk factors: A systematic review. Crit. Rev. Food Sci. Nutr. 2014, 54, 593-610. [CrossRef] [PubMed]

28. Esposito, K.; Marfella, R.; Ciotola, M.; Di Palo, C.; Giugliano, F.; Massimo D’Armiento, G.G.; Giugliano, D. Effect of a Mediterranean-style diet on endothelial dysfunction and markers of vascular inflammation in the metabolic syndrome. J. Am. Med. Assoc. 2004, 292, 1440. [CrossRef] [PubMed]

29. Calder, P.C.; Ahluwalia, N.; Brouns, F.; Buetler, T.; Clement, K.; Cunningham, K.; Marcos, A. Dietary factors and low-grade inflammation in relation to overweight and obesity. Br. J. Nutr. 2011, 106, S1-S78. [CrossRef] [PubMed]

30. Apovian, C.M. Obesity: Definition, comorbidities, causes, and burden. Am. J. Man Care 2016, 22, S176-S185.

31. Trichopoulou, A.; Costacou, T.; Bamia, C.; Trichopoulos, D. Adherence to a Mediterranean diet and survival in a Greek population. N. Engl. J. Med. 2003, 348, 2599-2608. [CrossRef] [PubMed] 
32. Sánchez-Villegas, A.; Martínez, J.A.; De Irala, J.; Martínez-González, M.A. Determinants of the adherence to an a priori defined Mediterranean dietary pattern. Eur. J. Nutr. 2002, 41, 249-257. [CrossRef] [PubMed]

33. Tur, J.A.; Romaguera, D.; Pons, A. Adherence to the Mediterranean dietary pattern among the population of the Balearic Islands. Br. J. Nutr. 2004, 92, 341-346. [CrossRef] [PubMed]

34. Fernández-Ballart, J.D.; Piñol, J.P.; Zazpe, I.; Corella, D.; Carrasco, P.; Toledo, E.; Martín-Moreno, J.M. Relative validity of a semi-quantitative food-frequency questionnaire in an elderly Mediterranean population of Spain. Br. J. Nutr. 2010, 103, 1808-1816. [CrossRef] [PubMed]

35. Moreiras, O.; Carbajal, A.; Cabrera, L.; Cuadrado, C. Tablas de Composición de Alimentos, 17th ed.; Piramide: Madrid, Spain, 2015.

36. Sureda, A.; Bibiloni, M.; Julibert, A.; Bouzas, C.; Argelich, E.; Llompart, I. Adherence to the Mediterranean siet and inflammatory markers. Nutrients 2018, 10, 62. [CrossRef] [PubMed]

37. Elosua, R.; Marrugat, J.; Molina, L.; Pons, S.; Pujol, E. Validation of the Minnesota leisure time physical activity questionnaire in Spanish men. The MARATHOM Investigators. Am. J. Epidemiol. 1994, 139, 1197-1209. [CrossRef] [PubMed]

38. Elosua, R.; Garcia, M.; Aguilar, A.; Molina, L.; Covas, M.I.; Marrugat, J. Validation of the Minnesota Leisure Time Physical Activity Questionnaire In Spanish Women. Investigators of the MARATDON Group. Med. Sci. Sports Exerc. 2000, 32, 1431-1437. [CrossRef] [PubMed]

39. Ruiz, A.C.; Pera, G.; Baena, J.D.; Mundet, X.T.; Alzamora, T.S.; Elosua, R.; Fàbrega, M.C. Validation of a spanish short version of the minnesota leisure time physical activity questionnaire (vrem). Revista Espanola Salud Publica 2012, 86, 495-508.

40. Martínez-Aldao, D.; Diz, J.C.; Varela, S.; Ayán, C. Analysis of the convergent validity of the Spanish short version of the Minnesota Leisure Time Physical Activity Questionnaire (VREM) and the Spanish version of the International Physical Activity Questionnaire in elderly people (IPAQ-E). Anales del Sistema Sanitario de Navarra 2019, 42, 147-157.

41. Sánchez-Lastra, M.A.; Martínez-Lemos, I.; Cancela, J.M.; Ayán, C. Physical activity questionnaires: A systematic review and analysis of their psychometric properties in Spanish population over 60 years old. Rev. Esp. Salud. Publica. 2018, 92, e201805018.

42. Byrne, N.M.; Hills, A.P.; Hunter, G.R.; Weinsier, R.L.; Schutz, Y. Metabolic equivalent: One size does not fit all. J. Appl. Physiol. 2005, 99, 1112-1119. [CrossRef]

43. Pate, R.R.; Pratt, M.; Blair, S.N.; Haskell, W.L.; Macera, C.A.; Bouchard, C.; Buchner, D.; Kriska, A. Physical activity and public health. J. Am. Med. Assoc. 1995, 273, 402. [CrossRef]

44. Ainsworth, B.E.; Haskell, W.L.; Whitt, M.C.; Irwin, M.L.; Swartz, A.M.; Strath, S.J.; Jacobs, D.R. Compendium of physical activities: An update of activity codes and MET intensities. Med. Sci. Sports Exerc. 2000, 32, S498-S516. [CrossRef] [PubMed]

45. Paffenbarger, R.S.; Wing, A.L.; Hyde, R.T. Physical activity as ana index of heart attack risk in college alumni. Am. J. Epidemiol. 1978, 108, 161-175. [CrossRef] [PubMed]

46. Sobejano Tornos, I.; Moreno Iribas, C.; Viñes Rueda, J.J.; Grijalba Uche, A.M.; Amézqueta Goñi, C.; Serrano Martínez, M. Population-based study of leisure time physical activity. Gac. Sanit. 2009, 23, 127-132. [CrossRef] [PubMed]

47. Blair, S.N.; Cheng, Y.; Holder, J.S. Is Physical Activity or Physical Fitness More Important in Defining Health Benefits? Med. Sci. Sports Exer. 2001, 33 (Suppl. S6), S379-S399. [CrossRef] [PubMed]

48. Lee, D.C.; Pate, R.R.; Lavie, C.J.; Sui, X.; Church, T.S.; Blair, S.N. Leisure-time running reduces all-cause and cardiovascular mortality risk. J. Am. Coll. Cardiol. 2014, 64, 472-481. [CrossRef] [PubMed]

49. Rosique-Esteban, N.; Díaz-López, A.; Martínez-González, M.A.; Corella, D.; Goday, A.; Martínez, J.A.; Tinahones, F. Leisure-time physical activity, sedentary behaviors, sleep, and cardiometabolic risk factors at baseline in the PREDIMED-PLUS intervention trial: A Cross-Sectional Analysis. PLoS ONE 2017, 12, e0172253. [CrossRef]

50. Hsueh, M.C.; Rutherford, R.; Huang, Y.H.; Chang Chien, H.Y.; Chang, C.H.; Park, J.H.; Liao, Y. Are older adults without a healthy diet less physically active and more sedentary? Nutrients 2019, 11, 1119. [CrossRef] [PubMed]

51. Da Silva, I.C.M.; Mielke, G.I.; Bertoldi, A.D.; Dourado Arrais, P.S.; Luiza, V.L.; Mengue, S.S.; PNAUM Working Group. Overall and leisure-time physical activity among Brazilian adults: National survey based on the global physical activity questionnaire. J. Phys. Act. Health 2018, 15, 212-218. [CrossRef] 
52. Schröder, H.; Marrugat, J.; Covas, M.; Elosua, R.; Pena, A.; Weinbrenner, T.; Masia, R. Population dietary habits and physical activity modification with age. Eur. J. Clin. Nutr. 2004, 58, 302-311. [CrossRef]

53. Gebel, K.; Ding, D.; Chey, T.; Stamatakis, E.; Brown, W.J.; Bauman, A.E. Effect of moderate to vigorous physical activity on all-cause mortality in middle-aged and alder Australians. JAMA Int. Med. 2015, 175, 970. [CrossRef] [PubMed]

54. Hidalgo-Santamaria, M.; Fernandez-Montero, A.; Martinez-Gonzalez, M.A.; Moreno-Galarraga, L.; Sanchez-Villegas, A.; Barrio-Lopez, M.T.; Bes-Rastrollo, M. Exercise intensity and incidence of metabolic syndrome: The SUN project. Am. J. Prev. Med. 2017, 52, 95-101. [CrossRef] [PubMed]

55. Rosengren, A.; Smyth, A.; Rangarajan, S.; Ramasundarahettige, C.; Bangdiwala, S.I.; Al-Habib, K.F.; Gupta, R. Socioeconomic status and risk of cardiovascular disease in 20 low-income, middle-income, and high-income countries: The prospective urban rural epidemiologic (PURE) study. Lancet Global. Health 2019, 7, e748-e760. [CrossRef]

56. Droomers, M.; Schrijvers, C.T.; Mackenbach, J.P. Educational level and decreases in leisure time physical activity: Predictors from the longitudinal GLOBE study. J. Epidemiol. Community Health 2001, 55, 562-568. [CrossRef] [PubMed]

57. Poirier, P.; Giles, T.D.; Bray, G.A.; Hong, Y.; Stern, J.S.; Pi-Sunyer, F.X.; Eckel, R.H. Obesity and cardiovascular disease: Pathophysiology, evaluation, and effect of weight loss. Circulation 2006, 113, 898-918. [CrossRef] [PubMed]

58. Mandviwala, T.; Khalid, U.; Deswal, A. Obesity and cardiovascular disease: A risk factor or a risk marker? Curr. Atheroscler. Rep. 2016, 18, 21. [CrossRef] [PubMed]

59. Du, H.; Bennett, D.; Li, L.; Whitlock, G.; Guo, Y.; Collins, R. Physical activity and sedentary leisure time and their associations with BMI, waist circumference, and percentage body fat in 0.5 million adults: The China Kadoorie Biobank study. Am. J. Clin. Nutr. 2013, 97, 487-496.

60. Tudor-Locke, C.; Burton, N.W.; Brown, W.J. Leisure-time physical activity and occupational sitting: Associations with steps/day and BMI in 54-59 year old Australian women. Prev. Med. 2009, 48, 64-68. [CrossRef]

61. Serra-Majem, L.; Bach, A.; Roman, B. Recognition of the Mediterranean diet: Going a step further. Public Health Nutr. 2006, 9, 101-102. [CrossRef]

62. Esposito, K.; Maiorino, M.I.; Bellastella, G.; Panagiotakos, D.B.; Giugliano, D. Mediterranean diet for type 2 diabetes: Cardiometabolic benefits. Endocrine 2017, 56, 27-32. [CrossRef]

63. D'Alessandro, A.; De Pergola, G. The Mediterranean diet: Its definition and evaluation of a priori dietary indexes in primary cardiovascular prevention. Int. J. Food Sci. Nutr. 2018, 69, 647-659. [CrossRef] [PubMed]

64. Di Daniele, N.; Noce, A.; Vidiri, M.F.; Moriconi, E.; Marrone, G.; Annicchiarico-Petruzzelli, M. Impact of Mediterranean diet on metabolic syndrome, cancer and longevity. Oncotarget 2017, 8, 8947-8979. [CrossRef] [PubMed]

65. Sadykova, K.; Shalkharova, Z.; Nuskabayeva, G.; Zhunissova, M.; Madenbay, K. Relationships between adherence to Mediterranean diet and components of the metabolic syndrome in the Kazakhstani population. Georgian Med. News 2018, 274, 97-102.

66. Tian, Y.; Jiang, C.; Wang, M.; Cai, R.; Zhang, Y.; He, Z. BMI, leisure-time Physical activity, and physical fitness in adults in China: Results from a series of national surveys, 2000-2014. Lancet Diab. Endocrinol. 2016, 4, 487-497. [CrossRef]

67. Arquer, A.; Elosua, R.; Covas, M.I.; Molina, L.; Marrugat, J. Amount and intensity of physical activity, fitness, and serum lipids in pre-menopausal women. Int. J. Sports Med. 2006, 27, 911-918. [CrossRef] [PubMed]

(C) 2019 by the authors. Licensee MDPI, Basel, Switzerland. This article is an open access article distributed under the terms and conditions of the Creative Commons Attribution (CC BY) license (http://creativecommons.org/licenses/by/4.0/). 